$R M x A C, \mathbf{5 3}, 225-234(2021)$

(c) 2021: Instituto de Astronomía, Universidad Nacional Autónoma de México

https://doi.org/10.22201/ia.14052059p.2021.53.42

\title{
FRONTIER RESEARCH IN ASTROPHYSICS IN THE GRAVITATIONAL WAVE ERA - I
}

\author{
Franco Giovannelli ${ }^{1}$ and Lola Sabau-Graziati ${ }^{2}$
}

\begin{abstract}
RESUMEN
En este artículo presentamos varios ejemplos que muestran la continua evolución en el conocimiento de la física de nuestro Universo. Se trata de una actualización del reciente articulo Giovannelli \& Sabau-Graziati (2019a). Una vez mas, queremos enfatizar que todos los objetos en nuestro Universo son interdependientes entre sí, y que las clasificaciones que habitualmente utilizamos se establecen de manera artificial para simplificar los problemas ya que la naturaleza evoluciona en todas sus manifestaciones con continuidad.
\end{abstract}

\section{ABSTRACT}

In this paper we will provide several examples that marked the continuous evolution on the knowledge of the physics of our Universe, updating our recent review (Giovannelli \& Sabau-Graziati, 2019a). We want to emphasize that all the objects in our Universe are interdependent on each other, and that the classifications - that are usually made to simplify problems - are artificial, since nature evolves in all its manifestations continuously.

Key Words: cosmology: theory — gamma ray bursts: general — gravitational waves — X-ray: binaries

\section{INTRODUCTION}

The Bridge Between the Big Bang and Biology undoubtedly exists (Giovannelli, 2001). Indeed, we are present here regardless of the origin of our Universe. So we must understand how to cross this bridge and understand what are the tools that allow us to make out the structure of the pillars supporting the bridge.

In order to cross this bridge, as always when we cross a bridge, we must advance slowly, step by step, with continuity, because everything is smoothly linked in the magma of the Universe: from the infinitely small to infinitely big, as discussed by Rees (1988).

In nature, nothing is isolated. Everything is related to the surrounding environment in a more or less strong way. However, the link exists. Fig 1 shows from left to right: i) a section of the metabolic network of a "simple" bacterium. Note that each point (each chemical compound) is connected to any other point through the complexity of the network (Luisi \& Capra, 2014); ii) the cosmic network: each point is connected to any other point through the complexity of the network (Credit: Andrew Pontzen/Fabio Gover-

\footnotetext{
${ }^{1}$ INAF - Istituto di Astrofisica e Planetologia Spaziali, Area di Ricerca di Roma-2, Via Fosso del Cavaliere, 100, I 00133 Roma, Italy (franco.giovannelli@inaf.it).

${ }^{2}$ INTA - Dpt de Cargas Utiles y Ciencias del Espacio Ctra de Ajalvir Km 4 - E 28850 Torrejón de Ardóz, Spain (sabaumd@inta.es).
}

nato, 2014; see also in (https://it.wikipedia. org/wiki/Cosmologiadelplasma). The large-scale structure of the Universe, as traced by the distribution of galaxies, is now being revealed by largevolume cosmological surveys. The structure is characterized by galaxies distributed along filaments, the filaments connecting in turn to form a percolating network. The objective of Shandarin, Habib \& Heitmann (2010) was to quantitatively specify the underlying mechanisms that drive the formation of the cosmic network. By combining percolation-based analyzes with N-body simulations of gravitational structure formation, they elucidate how the network has its origin in the properties of the initial density field (nature) and how its contrast is then amplified by the nonlinear mapping induced by the gravitational instability (nurture); iii) the human body network: each point (organ) is connected to any other point (organ) through the complexity of the network; iv) the human society network: each point is connected to any other point through the complexity of the network (Luisi \& Capra, 2014). The human population follows the cycle: birth, growth, aging, death. This is a general rule of the nature. Indeed also all the components of the Universe follow the same cycle. Therefore for a complete understanding of the history of the Universe it is necessary to search along that cycle.

In this paper - an updated version of our review recently published (Giovannelli \& Sabau-Graziati, 

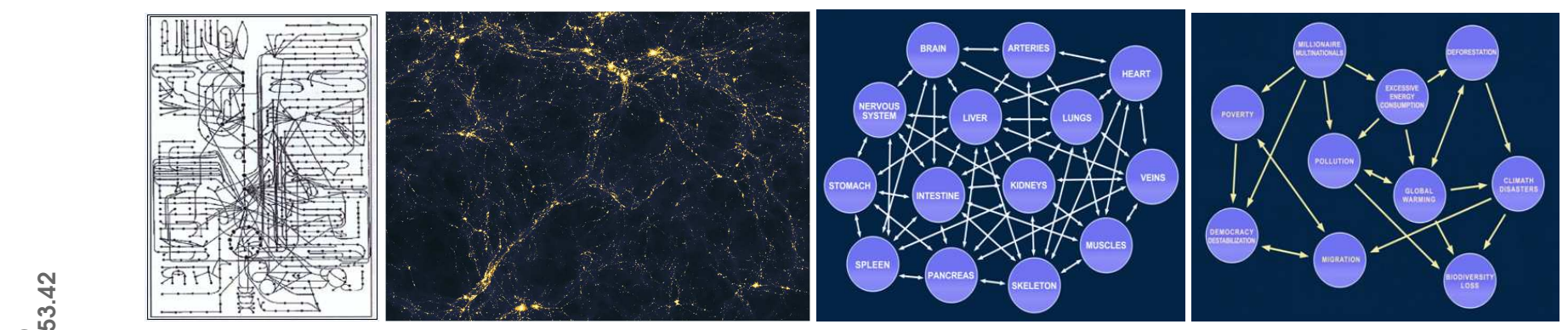

Fig. 1. From Left to Right: Section of the metabolic network of a "simple" bacterium (Luisi \& Capra, 2014), the "cosmic network" (Credit: Andrew Pontzen/Fabio Governato, 2014) see also in (https://it.wikipedia.org/wiki/ Cosmologiadelplasma), the human body network, and the human society network (Luisi \& Capra, 2014).

2019a) - we will jump most of the arguments discussed there, while we emphasize a few topics we consider important especially after the detection of the gravitational waves (GWs).

\subsection{Example of continuity in nature}

In the systems named cataclysmic variables (CVs), the accretion structure depends on the magnetic field of white dwarf (B) and on the transfer mass rate. Depending on B it is possible to classify $\mathrm{CVs}$ in three groups:

- Non Magnetic CVs (NMCVs): B $\sim 10^{4}-10^{6} \mathrm{G}$;

- Intermediate Polars (IPs): B $\sim 10^{6}-10^{7} \mathrm{G}$;

- Polars (MCVs): $\mathrm{B} \sim 10^{7}-10^{8} \mathrm{G}$.

However we have a smooth continuity among these classes, as discussed by Giovannelli \& SabauGraziati (2015).

Indeed, taking into account the average values of magnetic field intensity and orbital periods for polars and IPs, and the minimum and maximum value for both parameters ( $\mathrm{B}$ and $\mathrm{P}_{\text {orb }}$ ), it is possible to construct a very interesting plot (Fig. 2) that shows the evident continuity between the two classes of MCVs.

The nature in all its manifestations shows continuity. Then we have to abandon the "convenient method" of thinking everything in watertight compartments and to go toward a general model for compact accreting stars, like was done by Vladimir Lipunov and collaborators when they developed the "Scenario Machine" (Lipunov, 1987; Lipunov \& Postnov, 1988).

\section{THE PRESENT SITUATION ABOUT THE KNOWLEDGE OF THE PHYSICS OF OUR UNIVERSE}

Undoubtedly the advent of new generation experiments ground- and space-based have given a strong

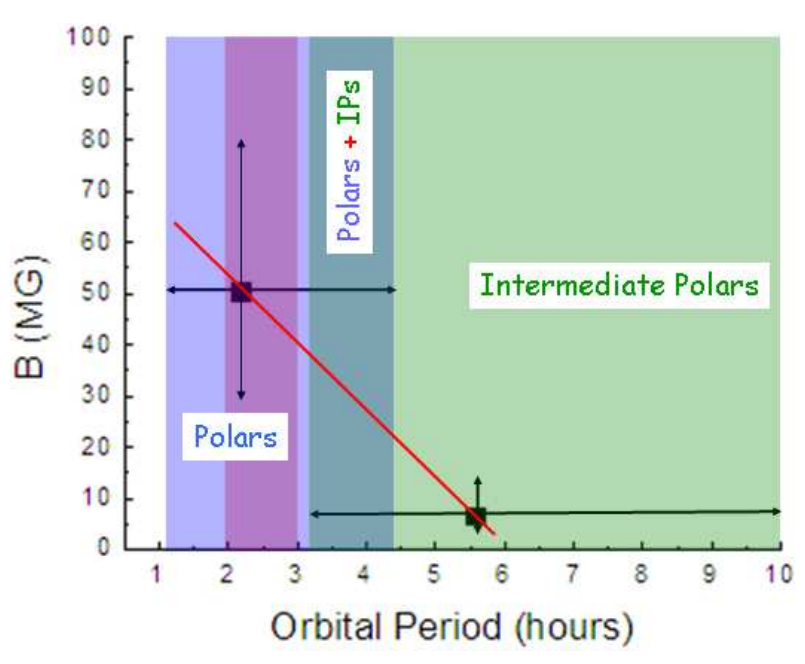

Fig. 2. Magnetic field intensity versus orbital period for MCVs. Polars and IPs are contained in the light blue and light green rectangles, respectively. Violet rectangle indicates the so-called "period gap". Cyan-50 rectangle represents the intersection between the Polars and IPs (adopted from Giovannelli \& Sabau-Graziati, 2015).

impulse for verifying current theories, and for providing new experimental inputs for developing a new physics for going, probably, over the standard model (SM). Recent results coming from Active Physics Experiments (APEs) and Passive Physics Experiments (PPEs) have opened such a new path.

The composition of the Universe is poorly known. Only $\sim 4.4 \%$ of ordinary matter, $\sim 0.6 \%$ of neutrinos, $\sim 22 \%$ of Dark Matter (DM), and $\sim 73 \%$ of dark Energy (DE). With the detection of GWEs a new window to the Universe has been opened.

An extensive review on the situation about the knowledge of the physics of our Universe has been recently published by Giovannelli \& Sabau-Graziati $(2016 ; 2019 a, b)$. The reader interested is invited to look at those papers. However, we are obliged to update a few topics that, in our opinion, could be useful 
for a better understanding of the open problems still existing in the modern astrophysics.

\subsection{Confirmation of the Theory of General Relativity}

In the last few years two further experimental results confirmed the validity of the theory of General Relativity (GR theory).

\subsubsection{Gravitational lenses}

Renn, Sauer \& Stachel (1997) published a historical reconstruction of some of Einstein's research notes dating back to 1912. These notes reveal that he explored the possibility of gravitational lensing 3 years before completing his general theory of relativity. On the basis of preliminary insights into this theory, Einstein had already derived the basic features of the lensing effect. When he finally published the very same results 24 years later, it was only in response to prodding by an amateur scientist.

Kochanek (2003) discussed "The whys and hows of finding 10,000 lenses", mentioning the first radio lens survey - the MIT - Green Bank survey (MG) - that found lenses by obtaining Very Large Array (VLA) snapshot images of flux-limited samples of $5 \mathrm{GHz}$ radio sources. The Hubble Space Telescope (HST), and Chandra observations (e.g. Dai \& Kochanek, 2005) showed without any doubt that the gravitational lensing is operating.

Gravitational lensing is widely and successfully used to study a range of astronomical phenomena, from individual objects, like galaxies and clusters, to the mass distribution on various scales, to the overall geometry of the Universe (Williams \& Schechter, 1997). They describe and assess the use of gravitational lensing as "gold standards" in addressing one of the fundamental problems in astronomy, the determination of the absolute distance scale to extragalactic objects, namely the Hubble constant.

Several papers have been published about the strong gravitational lensing (e.g. Tyson, Kochanski \& Dell'Antonio, 1998; Tyson, 2000 and references therein), and the weak gravitational lensing (Wittman et al., 2000). A review on "Gravitational Lenses" have been published by Blandford \& Kochanek (2004). A book on "Gravitational Lensing: Strong, Weak and Micro" was published by Meylan et al. (2006). Winn, Rusin \& Kochanek (2004) reported the most secure identification of a central image, based on radio observations of PMN J1632-0033.

Therefore, a further dowel supports the GR theory.
Considering that some divergent conclusions about cosmic acceleration were obtained using Type Ia supernovae (SNe Ia), with opposite assumptions on the intrinsic luminosity evolution, $\mathrm{Tu}, \mathrm{Hu} \&$ Wang (2019) use strong gravitational lensing systems to probe the cosmic acceleration. They found that the flat $\Lambda \mathrm{CDM}$ is strongly supported by the combination of the data sets from 152 strong gravitational lensing systems.

\subsubsection{Gravitational waves}

The Universe that contains by definition all the matter or all the energy available showed one important event that was possible to be detected on the Earth. This event was a further direct experimental demonstration of the validity of the GR theory. Indeed, on September 14, 2015 at 09:50:45 UTC the two detectors of the Laser Interferometer Gravitational-Wave Observatory (LIGO) simultaneously observed a transient gravitational-wave signal. It matches the waveform predicted by GR theory for the inspiral and merger of a pair of black holes and the ringdown of the resulting single black hole. The signal was observed with a significance $\geq 5.1 \sigma$. The source lies at a luminosity distance of $410_{-180}^{+160}$ Mpc corresponding to a redshift $\mathrm{z}=0.090_{-0.04}^{+0.03}$. In the source frame, the initial black hole masses are $36_{-4}^{+5} \mathrm{M}_{\odot}$ and $29 \pm 4 \mathrm{M}_{\odot}$, and the final black hole mass is $62 \pm 4 \mathrm{M}_{\odot}$ with $3.0 \pm 0.5 \mathrm{M}_{\odot} \mathrm{c}^{2}$ radiated in gravitational waves. All uncertainties define $90 \%$ credible intervals. These observations demonstrate the existence of binary stellar-mass black hole systems. This was the first direct detection of gravitational waves and the first observation of a binary black hole merger (Abbott et al., 2016a).

Abbott et al. (2016b) reported the second observation of a gravitational-wave signal produced by the coalescence of two stellar-mass black holes. The signal, GW151226, was observed by the twin detectors of the LIGO on December 26, 2015 at 03:38:53 UTC. The signal was detected at significance $\geq 5 \sigma$. The inferred source-frame initial black hole masses are $14.2_{-3.7}^{+8.3} \mathrm{M}_{\odot}$ and $7.5 \pm 2.3 \mathrm{M}_{\odot}$, and the final black hole mass is $20.8_{-1.7}^{+6.1} \mathrm{M}_{\odot}$. One finds that at least one of the component black holes has spin greater than 0.2 . This source is located at a luminosity distance of $440_{-190}^{+180} \mathrm{Mpc}$ corresponding to a redshift $\mathrm{z}$ $=0.09_{-0.04}^{+0.03}$. All uncertainties define a $90 \%$ credible interval. This second gravitational-wave observation provides improved constraints on stellar populations and on deviations from the GR theory.

For these detections of gravitational waves - first predicted by Einstein 100 years ago - Rainer Weiss, 
Barry Barish \& Kip Thorne have been awarded the 2017 Nobel prize in physics.

Abbott et al. (2016c) present a possible observing scenario for the Advanced LIGO (aLIGO) and Advanced Virgo gravitational-wave detectors over the next decade, with the intention of providing information to the astronomy community to facilitate planning for multimessenger astronomy with gravitational waves.

Gravitational waves provide a revolutionary tool to investigate yet unobserved astrophysical objects. Especially the first stars, which are believed to be more massive than present-day stars, might be indirectly observable via the merger of their compact remnants. An interesting paper by Hartwig et al. (2016) developed a self-consistent, cosmologically representative, semi-analytical model to simulate the formation of the first stars. They estimated the contribution of primordial stars to the merger rate density and to the detection rate of the aLIGO. Owing to their higher masses, the remnants of primordial stars produce strong GW signals, even if their contribution in number is relatively small. They found a probability of $\geq 1 \%$ that the current detection GW150914 is of primordial origin. The higher masses of the first stars boost their GW signal, and therefore their detection rate. Up to five detections per year with aLIGO at final design sensitivity originate from Pop III BH-BH mergers. Approximately once per decade, we should detect a BH-BH merger that can unambiguously be identified as a Pop III remnant.

On 2017 August 17 the merger of two compact objects with masses consistent with two neutron stars was discovered through gravitational-wave (GW170817), gamma-ray (GRB 170817A), and optical (SSS17a/AT2017gfo) observations. The optical source was associated with the early-type galaxy NGC 4993 at a distance of just $\sim 40 \mathrm{Mpc}$, consistent with the gravitational-wave measurement, and the merger was localized to be at a projected distance of $\sim 2 \mathrm{kpc}$ away from the galaxy's center (Abbott et al., 2017a,b).

Lipunov et al. (1995) predicted the NS-NS merger at a distance of $\leq 50 \mathrm{Mpc}$ and the possibility of detecting GWs!

This prediction was born by the "Scenario Machine" that describes the evolution of gravimagnetic rotators (Lipunov, 1987; Lipunov, \& Postnov, 1988), and recently commented by Giovannelli (2016).

On August 17, 2017 Multimessenger Astrophysics born! As pioneers of the Multifrequency Astrophysics, we are particularly happy!
Poggiani (2018) published an extensive review about the GW170817 event, in which she discussed also the related multimessenger observations.

The LIGO and Virgo interferometers have now confidently detected gravitational waves from a total of 10 stellar-mass binary black hole mergers and one merger of neutron stars, which are the dense, spherical remains of stellar explosions. Table 1 shows the eleven events (adapted from Abbott et al., 2019).

Barone et al. (1992) analyzed the class of CVs as sources of Gravitational Radiation, basing their analysis only on known objects at that time (168 CVs) taken from the Catalog of Ritter (1990).

From the analysis of GW emission from CVs, they derived that the emission frequencies are in the range $10^{-3}-10^{-5} \mathrm{~Hz}$ and that the GW flux at Earth is in the range $10^{-10}-10^{-13} \mathrm{erg} \mathrm{s}^{-1} \mathrm{~cm}^{-2}$ while the dimensionless amplitude is in the range $10^{-21}-10^{-23}$. These results constituted a solid basis for planning the construction of $\mathrm{GW}$ detectors (especially space-borne GW antennas). Moreover, these results provided the possibility of experimentally proving the effectiveness of the mechanism of Gravitational Radiation on CV evolution.

This important work was not sufficiently taken into account by the international community. However, now, after the detection of GWs coming from the fusion of black holes and neutron stars, the interest for that work has been rekindled in order to test the possibility of detecting GWs from CVs. Poggiani (2017), and the references therein) discussed this possibility, reaching the conclusion that $\mathrm{AM} \mathrm{CVn}$ systems and generally short-period systems are candidates for $\mathrm{GW}$ emission.

Amaro-Seoane et al. (2017) in response to the ESA call for L3 mission concepts, presented the Laser Interferometer Space Antenna (LISA) that since 2030 will allow to observe Gravitational Waves from cosmic sources, then to explore a Universe inaccessible otherwise, a Universe where gravity takes on new and extreme manifestations.

\subsection{Hubble Constant}

The Hubble constant $\left(\mathrm{H}_{0}\right)$ is one of the most important numbers in cosmology because it is needed to estimate the size and age of the universe. The important problem of determination of $\mathrm{H}_{0}$ value is one of the most exciting. Indeed, in the literature it is possible to find many determinations coming from different experiments using different methods. However, it is very complicated to obtain a true value for $\mathrm{H}_{0}$. It is necessary to have two measurements: i) spectroscopic observations that reveal the galaxy's 
TABLE 1

\section{SELECTED SOURCE PARAMETERS OF THE 11 CONFIDENT DETECTIONS. THE COLUMNS SHOW SOURCE-FRAME COMPONENT MASSES $\mathrm{M}_{1}$ AND $\mathrm{M}_{2}$, THE CHIRP MASS M, FINAL SOURCE-FRAME MASS $\mathrm{M}_{F}$, LUMINOSITY DISTANCE $\mathrm{D}_{L}$, AND REDSHIFT Z (ADAPTED FROM ABBOTT ET AL., 2019)}

\begin{tabular}{|c|c|c|c|c|c|c|}
\hline $\begin{array}{l}\text { GW Event } \\
\text { (name) }\end{array}$ & $\begin{array}{l}\mathrm{m}_{1} \\
\left(\mathrm{M}_{\odot}\right) \\
\end{array}$ & $\begin{array}{l}\mathrm{m}_{2} \\
\left(\mathrm{M}_{\odot}\right) \\
\end{array}$ & $\begin{array}{l}\mathrm{M} \\
\left(\mathrm{M}_{\odot}\right) \\
\end{array}$ & $\begin{array}{l}\mathrm{M}_{\mathrm{f}} \\
\left(\mathrm{M}_{\odot}\right) \\
\end{array}$ & $\begin{array}{l}d_{L} \\
(\mathrm{Mpc})\end{array}$ & $\begin{array}{l}z \\
\text { (redshift) }\end{array}$ \\
\hline GW 150914 & $35.6_{-3.1}^{+4.7}$ & $30.6_{-4.4}^{+3.0}$ & $28.6_{-1.5}^{+1.7}$ & $63.1_{-3.0}^{+3.4}$ & $440_{-170}^{+150}$ & $0.09_{-0.03}^{+0.03}$ \\
\hline GW 151012 & $23.2_{-5.5}^{+14.9}$ & $13.6_{-4.8}^{+4.1}$ & $15.2_{-1.2}^{+2.1}$ & $35.6_{-3.8}^{+10.8}$ & $1080_{-490}^{+550}$ & $0.21_{-0.09}^{+0.09}$ \\
\hline GW 151226 & $13.7_{-3.2}^{+8.8}$ & $7.7_{-2.5}^{+2.2}$ & $8.9_{-0.3}^{+0.3}$ & $20.5_{-1.5}^{+6.4}$ & $450_{-190}^{+180}$ & $0.09_{-0.04}^{+0.04}$ \\
\hline GW 170104 & $30.8_{-5.6}^{+7.3}$ & $20.0_{-4.6}^{+4.9}$ & $21.4_{-1.8}^{+2.2}$ & $48.9_{-4.0}^{+5.1}$ & $990_{-430}^{+440}$ & $0.20_{-0.08}^{+0.08}$ \\
\hline GW 170608 & $11.0_{-1.7}^{+5.5}$ & $7.6_{-2.2}^{+1.4}$ & $7.9_{-0.2}^{+0.2}$ & $17.8_{-0.7}^{+3.4}$ & $320_{-110}^{+120}$ & $0.07_{-0.02}^{+0.02}$ \\
\hline GW 170729 & $50.2_{-10.2}^{+16.2}$ & $34.0_{-10.1}^{+9.1}$ & $35.4_{-4.8}^{+6.5}$ & $79.5_{-10.2}^{+14.7}$ & $2840_{-1360}^{+1400}$ & $0.49_{-0.21}^{+0.19}$ \\
\hline GW 170809 & $35.0_{-5.9}^{+8.3}$ & $23.8_{-5.2}^{+5.1}$ & $24.9_{-2.7}^{+2.1}$ & $56.3_{-3.8}^{+5.2}$ & $1030_{-390}^{+320}$ & $0.20_{-0.07}^{+0.05}$ \\
\hline GW 170814 & $30.6_{-3.0}^{+5.6}$ & $25.2_{-4,0}^{+2,8}$ & $24.1_{-1.1}^{+1.4}$ & $53.2_{-2.4}^{+3.2}$ & $600_{-220}^{+150}$ & $0.12_{-0.04}^{+0.03}$ \\
\hline GW 170817 & $1.46_{-0.10}^{+0.12}$ & $1.27_{-0.09}^{+0.09}$ & $1.186_{-0.001}^{+0.001}$ & $\leq 2.8$ & $40_{-15}^{+7}$ & $0.01_{-0.00}^{+0.00}$ \\
\hline GW 170818 & $35.4_{-4.7}^{+7.5}$ & $26.7_{-5.2}^{+4.3}$ & $26.5_{-1.7}^{+2.1}$ & $59.4_{-3.8}^{+4.9}$ & $1060_{-380}^{+420}$ & $0.21_{-0.07}^{+0.07}$ \\
\hline GW 170823 & $39.5_{-6.7}^{+11.2}$ & $29.0_{-7.8}^{+6.7}$ & $29.2_{-3.6}^{+4.6}$ & $65.4_{-7.4}^{+10.1}$ & $1940_{-900}^{+970}$ & $0.35_{-0.16}^{+0.15}$ \\
\hline
\end{tabular}

redshift, indicating its radial velocity; ii) the galaxy's precise distance from Earth (and this is the most difficult value to determine).

A large summary about the methods used for $\mathrm{H}_{0}$ determination, and its derived values can be found in the Proceedings of the Fall 2004 Astronomy 233 Symposium on "Measurements of the Hubble constant" (Damon et al., 2004). In this book, Teymourian (2004), after a comparison of many constraints on the Hubble constant determinations, reports a value $\mathrm{H}_{0}=68 \pm 6 \mathrm{~km} \mathrm{~s}^{-1} \mathrm{Mpc}^{-1}$.

A discussion about the Hubble constant has been published by Giovannelli \& Sabau-Graziati (2014, $2019 \mathrm{~b}$ ), where it is possible to find also a large number of references, reporting the many controversial evaluations of $\mathrm{H}_{0}$.

Figure 6 shows the determinations of $\mathrm{H}_{0}$ since 1970 (adapted from John Huchra, 2008). Practically all the determinations lie in the range $40-100 \mathrm{~km} \mathrm{~s}^{-1}$ $\mathrm{Mpc}^{-1}$ (marked with light-blue rectangle), and most of them are converging in the range $55-70 \mathrm{~km} \mathrm{~s}^{-1}$ $\mathrm{Mpc}^{-1}$ (marked with light-red rectangle).

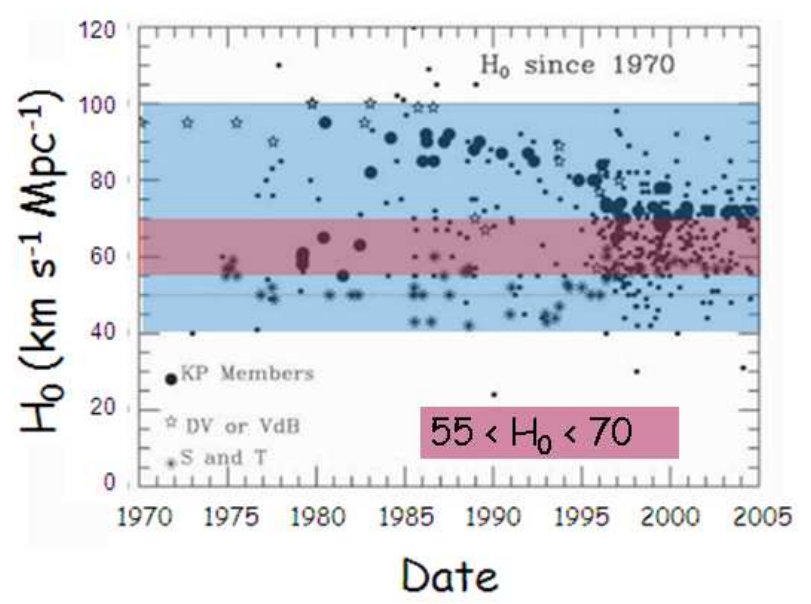

Fig. 3. The Hubble constant determinations since 1970. The light-blue rectangle limits all the $\mathrm{H}_{0}$ determinations. The light-red rectangle shows the narrow limits to which the values of $\mathrm{H}_{0}$ are converging (Giovannelli \& SabauGraziati (2016) after John Huchra, 2008). 
John Huchra (2010) listed the last updated collection of data on October 7, 2010, just one day before his sudden death (Huchra, 2010 in https:// www.cfa.harvard.edu/ dfabricant/huchra/hubble. plot.dat). ${ }^{(*)}$

(*) Professor John Huchra, died unexpectedly October 8th, 2010.

The CMB is used to predict the current expansion rate of the universe by best-fitting cosmological model. At low redshift baryon acoustic oscillation (BAO) measurements have been used - although they cannot independently determine $\mathrm{H}_{0}$ - for constraining possible solutions and checks on cosmic consistency. Comparing these measurements they found $\mathrm{H}_{0}=69.6 \pm 0.7 \mathrm{~km} \mathrm{~s}^{-1} \mathrm{Mpc}^{-1}$ (Bennett et al., 2014).

Does this determination, finally, close the history about the search of the "true" value of $\mathrm{H}_{0}$ ?

Independent estimation of the Hubble constant from the luminosity distance of GW signal (GW 170817) and the event association with NGC 4993 (Abbott et al., 2017c) gives a value $\mathrm{H}_{0}=70.0_{-8.0}^{+12.0}$ $\mathrm{km} \mathrm{s}^{-1} \mathrm{Mpc}^{-1}$.

However, due to large errors, this value of Hubble constant do not add any significative information, but being obtained with independent methods provide a good support for the value of $\mathrm{H}_{0}=69.6$ $\pm 0.7 \mathrm{~km} \mathrm{~s}^{-1} \mathrm{Mpc}^{-1}$, determined by Bennett et al. (2014).

\subsection{Reionization Epoch}

The formation of the first stars and quasars marks the transformation of the universe from its smooth initial state to its clumpy current state. In current cosmological models, the first sources of light began to form at a redshift $\mathrm{z} \sim 30$ and reionized most of the hydrogen in the universe by $\mathrm{z} \sim 7$ (see review by Loeb \& Barkana, 2001).

Figure 4 shows schematically the updated experimental situation about cosmic sources (galaxies, GRBs, QSOs, SNe) detected at high redshifts. The light-red rectangle marks the possible range of $\mathrm{z}$ during which the reionization occurred.

However, although there is rather good agreement about the epoch of reionization, how really reionization occurs is still object of debate. Indeed, Dopita et al. (2011), considering that observations show that the measured rates of star formation in the early universe are insufficient to produce reionization, suggest the presence of another source of ionizing photons. This source could be the fast accretion

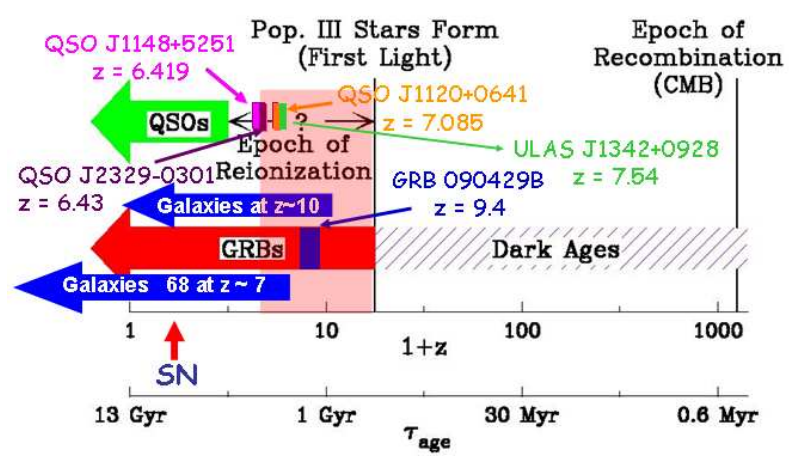

Fig. 4. A sketch of reionization epoch (after Xiangping Wu's Talk at the Summer School on "Cosmic Reionization" at the KIAA-PKU , Beijing, China, July 1-11, 2008).

shocks formed around the cores of the most massive haloes.

A deep discussion about the reionization epoch has been reported in the review paper by Giovannelli \& Sabau-Graziati (2019b, and the referees therein).

An interesting review about The epoch of reionization was published by Zaroubi (2013). Recently An Introductory Review on Cosmic Reionization have been published by Wise (2019).

Recently, Yang et al. (2019) announced the discovery of six new $\mathrm{z} \gtrsim 6.5$ quasars.

This work opens a glimmer of light on the possibility of revealing in the future, with the advent of JWST, the presence of quasars immediately after the formation of the first Pop. III stars at $\mathrm{z} \approx 25$, as well as the possibility of detecting GRBs up to that redshift (Lamb \& Reichart, 2000; Ciardi \& Loeb, 2000; Bromm \& Loeb, 2002). Indeed, the detection of the GRB 090429B at $\mathrm{z} \simeq 9.4$ (Cucchiara et al., 2011) is a good omen to think that future experiments can reveal GRBs up to the fateful threshold of $\mathrm{z} \approx 25$.

\subsection{Gamma Ray Bursts}

Long discussions about Gamma-ray bursts (GRBs) can be found in numerous publications. A list of these can be found in GSG2004 and in Giovannelli \& Sabau-Graziati (2016, 2019a,b).

Although big progress has been obtained in the last few years, GRBs theory needs further investigation in the light of the experimental data coming from old and new satellites, often coordinated, such as BeppoSAX or BATSE/RXTE or ASM/RXTE or IPN or HETE or INTEGRAL or SWIFT or AGILE or FERMI or MAXI.

The idea that GRBs could be associated to gravitational waves (GWs) emission is now popular. Indeed, short GRBs are believed to be produced by 
the mergers of either double NSs or NS-BH binaries (Nakar, 2007) and the observation of a kilonova associated with GRB130603B (Tanvir et al., 2013; Berger, Fong \& Chornock, 2013) lends support to this hypothesis.

Thanks to the NASA's Swift satellite we assisted to ten years of amazing discoveries in time domain astronomy. Its primary mission is to chase GRBs. The list of major discoveries in GRBs and other transients includes the long-lived X-ray afterglows and flares from GRBs, the first accurate localization of short GRBs, the discovery of GRBs at high redshift $(\mathrm{z}>8)$ (Gehrels \& Cannizzo, 2015). And essentially thanks to these discoveries we are now closer to understand the real nature of GRBs.

\subsection{Anomalous X-ray Pulsars and Soft Gamma Repeaters: Magnetars}

Since their discovery, neutron stars (NSs) have excited a broad range of interests not only in the astrophysical context, but also in terms of fundamental physics.

NSs are characterized by extreme conditions, such as dense matter, rapid rotation, and high magnetic field, they have proved to be ideal laboratories to test fundamental physics, which cannot be achieved by ground-based experiments.

Multi-wavelength observations from radio to the highest energy gamma-rays have revealed a remarkable diversity of NSs (Kaspi, 2010).

In the last two decades a new class of X-ray binaries has been recognized. They are X-ray pulsars with properties clearly different from those of the common HMXBs. This new group of pulsars constitutes a subclass of the LMXBs, characterized by lower luminosities, higher magnetic fields and smaller ages than non-pulsating LMXBs. These objects have been called Anomalous X-ray Pulsars (AXPs) (e.g. GSG2004, and the references therein) and this is now the current accepted name. Soon after their discovery, this new class of objects, whose nature was recognized to be that of neutron stars, were characterized by a spin periods ranging between $5.5-11.8 \mathrm{~s}-$ and $\dot{\mathrm{P}}$, in the range $0.05-10 \times 10^{-11}$ $\mathrm{s} \mathrm{s}^{-1}$ - contrary to the larger spread of those of HMXBs $\left(0.069-\right.$ few $\left.\times 10^{3} \mathrm{~s}\right)$. Spin periods of AXPs are monotonically increasing on timescales of $\sim 10^{4}$ $4 \times 10^{5} \mathrm{yr}$.

Measurements of the spin down rates of SGRs and AXPs have been interpreted as evidence of very strong magnetic fields at the collapsed object poles, roughly two orders of magnitude greater than those of the 'normal' X-ray pulsars. For this reason they are now known as 'magnetars'. Their derived magnetic field intensity is $\sim 10^{14}-10^{15} \mathrm{G}$.

The problem of the nature of magnetars is one of the hottest in modern astrophysics. Indeed, for instance, Dar (2003) argued that, instead, the observations support the hypothesis that SGRs and AXPs are neutron stars that have suffered a transition into a denser form of nuclear matter to become, presumably, strange stars or quark stars. Internal heat and slow gravitational contraction long after this transition can power both their quiescent X-ray emission and their star quakes, which produce 'soft' gamma ray bursts. Dar (2006) discussed once more this idea by using results from short-duration hardspectrum GRBs, such as 050509B, 050709, 050724, and 050813 , which could have been the narrowly beamed initial spike of hyperflares of SGRs in galaxies at cosmological distances. Such bursts are expected if SGRs are young hyperstars, i.e. neutron stars where a considerable fraction of their neutrons have converted to hyperons and/or strange quark matter. Ghosh (2009) discussed some of the developments in the quark star physics along with the consequences of possible hadron to quark phase transition at high density scenario of neutron stars and their implications on the Astroparticle Physics.

However, the nature of magnetar is not yet definitively proved. Giovannelli \& Sabau-Graziati (2006) speculated as follows: if magnetic fields of $\sim 10^{15} \mathrm{G}$ can be expected in order to explain the behaviour of magnetars, an almost 'obvious' consequence can be derived from the diagram magnetic field intensity versus the dimension of the relative cosmic source. They extrapolated the value of B up to $10^{15} \mathrm{G}$; the correspondent dimension of the source is of $\sim 10 \mathrm{~m}$. This could be the dimension of the acceleration zone in a supercompact star, probably a quark star. If you construct a trap, the rat falls into it!

Table 2 shows the pulse timing properties of magnetars, the derived magnetic field intensity, the age (after Olausen \& Kaspi, 2014 and Kaspi \& Beloborodov, 2017), and their association with SNRs (after Giovannelli \& Sabau-Graziati, 2006).

The open questions about magnetars are numerous, namely: i) What are the distances of the Galactic magnetars? Then what is the Energetics? ii) What is the number-intensity relation for giant magnetar flares? iii) What are the SGR and AXP birth rate? What are their lifetimes? How many SGRs and AXPs are in the Milky Way? iv) What kind of supernova produces a SGR or an AXP? v) What is the relation between SGRs and AXPs? Does one evolve into the other, or are they separate manifes- 
TABLE 2

PULSE TIMING PROPERTIES OF MAGNETARS AND MAGNETAR CANDIDATES (AFTER OLAUSEN \& KASPI, 2014 AND KASPI \& BELOBORODOV, 2017)

\begin{tabular}{|c|c|c|c|c|}
\hline $\begin{array}{c}\text { AXP \& SGR (Magnetars) } \\
\text { (name) }\end{array}$ & $\begin{array}{l}P_{\text {pulse }} \\
\text { (s) }\end{array}$ & $\begin{array}{c}\dot{P}_{\text {pulse }} \\
10^{-11} \mathrm{~s} \mathrm{~s}^{-1}\end{array}$ & $\begin{array}{c}\mathrm{B} \\
\left(10^{14} \mathrm{G}\right)\end{array}$ & $\begin{array}{l}\text { Spin-down } \\
\text { Age (kyr) }\end{array}$ \\
\hline CXOU J0110-721 (SMC) & 8.02 & 1.88 & 3.9 & 6.8 \\
\hline $4 \mathrm{U} 0142+614$ & 8.69 & 0.20 & 1.3 & 68 \\
\hline SGR $0418+5729$ & 9.08 & 0.0004 & 0.061 & 36000 \\
\hline SGR $0501+4516$ & 5.76 & 0.582 & 1.9 & 16 \\
\hline SGR 0526-66 (N49) & 8.05 & 3.8 & 5.6 & 3.4 \\
\hline 1E 1048.1-5937 & 6.46 & $\sim 2.25$ & 3.9 & 4.5 \\
\hline PSR J1119-6127 & 0.41 & - & 4.1 & 1.6 \\
\hline 1E 1547.0-5408 & 2.07 & $\sim 4.77$ & 3.2 & 0.69 \\
\hline PSR J1622-4950 & 4.33 & 1.7 & 2.7 & 4.0 \\
\hline SGR 1627-41 (G337.0-0.1) & 2.59 & 1.9 & 2.2 & 2.2 \\
\hline CXOU J164710.2-455216 & 10.61 & $<0.4$ & $<0.66$ & $>420$ \\
\hline 1RXS J170849.0-400910 & 11.00 & 1.91 & 4.6 & 9.1 \\
\hline CXOU J171405.7-381031 & 3.82 & 6.40 & 5.0 & 0.95 \\
\hline SGR J1745-2900 & 3.76 & 0.66 & 1.6 & 9.0 \\
\hline SGR 1806-20 (G10.0-0.3) & 7.54 & $\sim 49.5$ & 20 & 0.24 \\
\hline XTE J1810-197 & 5.54 & 0.78 & 2.1 & 11 \\
\hline Swift J1822.3-1606 & 8.44 & 0.031 & 0.51 & 440 \\
\hline SGR 1833-0832 & 7.56 & 0.35 & 1.6 & 34 \\
\hline Swift J1834.9-0846 & 2.48 & 0.80 & 1,4 & 4.9 \\
\hline 1E1841-045 (Kes 73) & 11.78 & 3.93 & 6.9 & 4.7 \\
\hline PSR J1846-0258 & 0.33 & - & 0.49 & 0.73 \\
\hline 3XMM J185246.6+003317 & 11.56 & - & $<0.41$ & $>1300$ \\
\hline SGR $1900+14(G 42.8+0.6)$ & 5.2 & 9.2 & 7.0 & 0.90 \\
\hline SGR $1935+2154$ & 3.24 & - & 2.2 & 3.6 \\
\hline 1E2259+586 (CTB 109) & 6.98 & 0.048 & 0.59 & 230 \\
\hline \multicolumn{5}{|l|}{ Magnetar Candidates } \\
\hline SGR 0755-2933 & - & - & - & - \\
\hline SGR 1801-23 & - & - & - & - \\
\hline SGR 1808-20 & - & - & - & - \\
\hline AX J 1818.8-1559 & - & - & - & - \\
\hline AX J1845-026(G29.6+586) & 6.97 & - & - & - \\
\hline SGR $2013+34$ & - & - & - & - \\
\hline
\end{tabular}

tations of magnetars? vi) Are really the collapsed objects in SGRs and AXPs neutron stars? Alternatively, could they be quark stars? vii) How many other manifestations of magnetars exist?

In order to answer to these open questions, more sensitive instruments, more detailed theories, and more data (probably in the next 30 years) are necessary.

In the extensive and excellent reviews by Kitamoto et al. (2014) and by Kaspi \& Beloborodov (2017) most of the critical points about magnetars have been deeply discussed.

A large diversity of neutron stars has been discovered by multifrequency observations from the radio band to the X-ray and gamma-ray energy ranges. Among different manifestation of neutron stars - which include SGRs, AXPs, high-B pulsars (HBPs), high-E binaries (HEBs), rotating radio transients (RRATs), central compact objects (CCOs), rotation-powered radio pulsars (RPPs), and X-ray isolated neutron stars (XINSs) (Harding, 2013) - magnetars are the strongest magnetized objects.

These various manifestations of neutron stars show different characteristics of rotation period $\mathrm{P}$ and its derivative $\dot{\mathrm{P}}$. The measurements of $\mathrm{P}$ and $\dot{\mathrm{P}}$ provide to estimate the dipole magnetic field strength $\mathrm{B}_{\mathrm{d}} \propto \sqrt{\mathrm{P} \dot{\mathrm{P}}}$ and characteristic age $\tau_{\mathrm{c}}=$ $\mathrm{P} / 2 \dot{\mathrm{P}}$.

Figure 5 shows the $\mathrm{P}-\dot{\mathrm{P}}$ diagram (Enoto, 2018), where SGRs and AXPs are collectively called "magnetars" since their slow rotation $(\mathrm{P} \sim 2-12 \mathrm{~s})-$ 


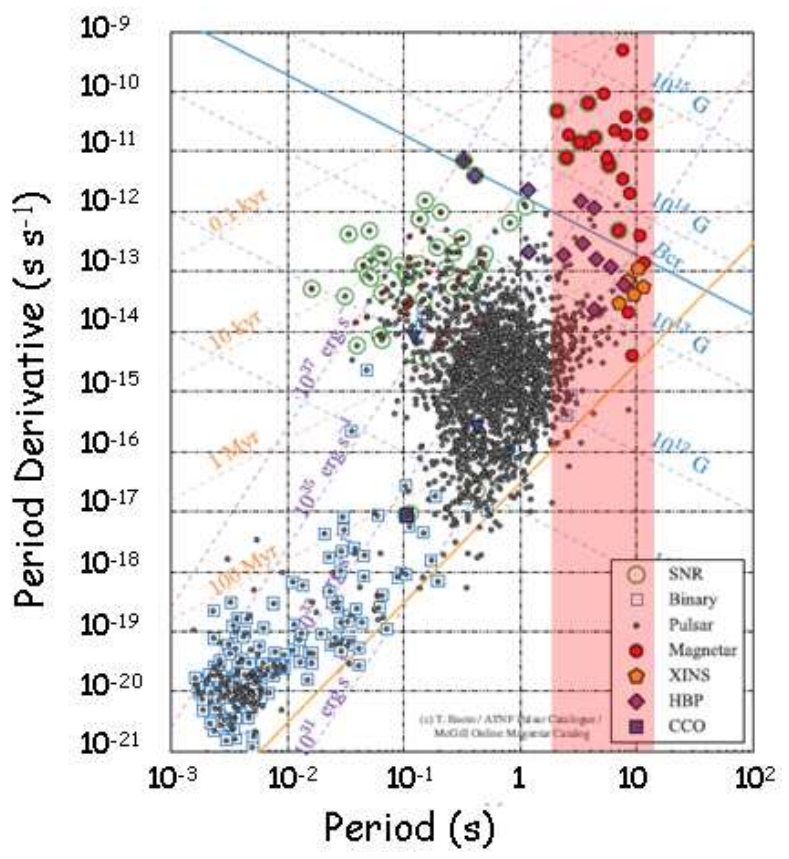

Fig. 5. Magnetars and neutron stars on the $\mathrm{P}-\dot{\mathrm{P}}$ diagram. The light-red rectangle limits the range of magnetars periods (adapted from Enoto, 2018).

with the exception of PSR J1119-6127 ( $\mathrm{P}=0.41 \mathrm{~s})$ and PSR J1846-0258 $(\mathrm{P}=0.33 \mathrm{~s})$ - and high period derivatives $\left(\dot{\mathrm{P}} \sim 10^{-13}-10^{-9} \mathrm{~s} \mathrm{~s}^{-1}\right)$ indicate high magnetic fields $\mathrm{B}=10^{14-15} \mathrm{G}$ and young characteristic age $\tau_{\mathrm{c}} \lesssim 10-100 \mathrm{kyr}$. To date, there are 29 known magnetars in the Milky Way and local universe (see Table 2).

It seems almost natural to think about a continuity among different classes of neutron star systems. However radio pulsations that have been observed from about 2000 neutron stars with weaker magnetic fields have never been detected from any of the known magnetars until the paper by Camilo et al. (2006) which showed that XTE J1810-197 - the first transient magnetar discovered (Ibrahim et al., 2004) — emits bright, narrow, highly linearly polarized radio pulses, observed at every rotation, thereby establishing that magnetars can be radio pulsars. Thus, these observations which link magnetars to ordinary radio pulsars, rule out alternative accretion models for AXPs, and provide a new window into the coronae of magnetars.

In the excellent review paper by Kaspi \& Beloborodov (2017) most of the critical points about magnetars have been deeply discussed. They concluded that: "The magnetar model has now been used to predict, naturally and uniquely, a wide va- riety of remarkable phenomena and behaviors in sources that once seemed highly anomalous. The now seamless chain of phenomenology from otherwise conventional radio pulsars through sources previously known for radically different behavior makes clear that these objects are one continuous family, with activity correlated with spin-inferred magnetic field strength. Recent advances in the physics of these objects, from the core through the crust and to the outer magnetosphere, hold significant promise".

\section{CONCLUSIONS}

It is difficult to summarize the conclusions in a few words. We simply want to emphasize that all the objects in our Universe are interdependent on each other (as shown in Fig. 1), and that the classifications - that are usually made to simplify problems - are artificial, since nature evolves in all its manifestations continuously, as demonstrated with the examples of CVs and neutron star systems.

Acknowledgments: This research has made use of "The NASA's Astrophysics Data System".

\section{REFERENCES}

Abbott, B. P., Abbott, R., Abbott, T. D., et al. 2016a, PhRvL, 116, 1102

Abbott, B. P., et al. 2016b, PhRvL, 116, 1103 2016c, LRR, 19, 1 2017a, PhRvL, 119, 161101 2017b, ApJL, 848, 12

Abbott, B. P., Abbott, R., Abbott, T. D., et al. 2017c, Natur, 551, 85 2019, PhRvX, 9, 031040

Amaro-Seoane, P., et al. 2017, arXiv:1702.00786

Barone, F., et al. 1992, GReGr, 24, 323

Bennett, C. L., et al. 2014, ApJ, 794, 135

Berger, E., Fong, W., \& Chornock, R. 2013, ApJL, 774, 23

Blandford, R. D. \& Kochanek, C. S. 2004, in Dark Matter in the Universe (Second Edition), ed. Bahcall, J., et al., World Scientific Publishing Co. Pte. Ltd., ISBN 9789812567185, 103

Bromm, V. \& Loeb, A. 2002, ApJ, 575, 111

Camilo, F., et al. 2006, Natur, 442, 892

Ciardi, B. \& Loeb, A. 2000, ApJ, 540, 687

Cucchiara, A., et al. 2011, ApJ, 736, 7

Dai, X. \& Kochanek, C. S. 2005, ApJ, 625, 633

Damon, E., et al. 2004, Proc. of the Fall 2004 Astronomy 233 Symposium on Measurements of the Hubble Constant, ed. Campbell, D. B. \& Deneva, J., 61

Dar, A. 2003, in Frontier Objects in Astrophysics and Particle Physics, ed. Giovannelli, F. \& Mannocchi, G. SIF 85, 253 . 2006, ChJA\&A Supp., 6, 323 
Dopita, M. A., et al. 2011, Ap\&SS, 335, 345

Enoto, T. 2018, in Quarks and Compact Stars 2017 (QCS2017), JPS Conf. Proc., id.011046, 7

Gehrels, N. \& Cannizzo, J. K. 2015, JHEAp, 7, 2

Ghosh, S. K. 2009, in Frontier Objects in Astrophysics and Particle Physics, ed. Giovannelli, F. \& Mannocchi, G., SIF, Bologna, Italy, 98, 243

Giovannelli, F. 2001, The bridge between the big bang and biology: stars, planetary systems, atmospheres, volcanoes: their link to life, ed. Giovannelli, F., CNR: President's Bureau of the CNR, Mario Apice (General Coordinator), 1 2016, in High Energy Astrophysics in Southern Africa (HEASA 2016). Online at http://pos. sissa.it/cgi-bin/reader/conf . cgi? confid=275, id. 31

Giovannelli, F. \& Sabau-Graziati, L. 2004, SSR, 112, 1 2006, ChJAS, 6, 1

2014, in Multifrequency Behaviour of High Energy Cosmic Sources - X, Acta Polytechnica CTU Proceedings, 1(1), 1

2015, The Golden Age of Cataclysmic Variables and Related Objects - III, Online at http://pos.sissa.it/cgi-bin/reader/conf.cgi? confid=255, id. 1 2016, in Frontier Research in Astrophysics II, Online at https://pos.sissa.it/cgi-bin/reader/ conf . cgi? confid=269, id. 1 2019a, RMxAC, 51, 66

2019b, in Multifrequency Behaviour of High Energy Cosmic Sources - XIII, Pos-SISSA (in press)

Harding, A. K. 2013, Front. Phys., 8, 6, 679

Hartwig, T., et al. 2016, MNRAS, 460, 74

Huchra, J. 2008, https://www.cfa.harvard.edu/ $\sim$ dfabricant/huchra/hubble/

2010, https://www.cfa.harvard.edu/ $\sim$ dfabricant/huchra/hubble.plot.dat

Ibrahim, A. I., et al. 2004, ApJ, 609, 21

Kaspi, V. M. 2010, PNAS, 107, 7147

Kaspi, V. M. \& Beloborodov, A. M. 2017, ARA\&A, 55, 261

Kitamoto, S., et al. 2014, arXiv:1412.1165

Kochanek, C. S. 2003, ASPC, 291, 245

Lamb, D. Q. \& Reichart, D. E. 2000, ApJ, 536, 1

Lipunov, V. M. 1987, Ap\&SS, 132, 1 1995, in Frontier Objects in Astrophysics and
Particle Physics, ed. Giovannelli, F. \& Mannocchi, G., SIF, Bologna, Italy, 47, 61

Lipunov, V. M. \& Postnov, K. A. 1988, Ap\&SS, 145, 1

Loeb, A. \& Barkana, R. 2001, ARA\&A, 39, 19

Luisi, P. L. \& Capra, F. 2014, The systems View of Life, Cambridge Univ. Press, 510

Meylan, G., et al. 2006, Gravitational lensing: strong, weak and micro, ed. Meylan, G., Jetzer, P., \& North, P., Berlin: Springer, ISBN 3-540-30309-X, ISBN 9783-540-30309-1, XIII + 552

Nakar, E. 2007, Phys. Rep., 442, 166

Olausen, S. A. \& Kaspi, V. M. 2014a, ApJS, 212, 6

Poggiani, R. 2017, in The Golden Age of Cataclysmic Variables and Related Objects - IV, online at https://pos.sissa.it/cgi-bin/chairman/ chlist. cgi? confid=315, id. 008 2018, in Frontier Researc in Astrophysics - III, online at https://pos.sissa.it/cgi-bin/ chairman/chlist.cgi? confid=331, id. 013

Rees, M. J. 1988, in Origins, ed. Fabian, A.C., Cambridge Univ. Press, 1

Renn, J., Sauer, T., \& Stachel, J. 1997, Science, 275, 184

Ritter, H. 1990, A\&AS, 85, 1179

Shandarin, S., Habib, S., \& Heitmann, K. 2010, PhRvD, 81,103006

Tanvir, N. R., Levan, A. J., Fruchter, A. S., et al. 2013, Natur, 500, 547

Teymourian, A. 2004, in Proc. of the Fall 2004 Astronomy 233 Symp. on Measurements of the Hubble Constant, ed. Campbell, D. B. \& Deneva, J., 55

Tu, Z. L., Hu, J., \& Wang, F. Y. 2019, MNRAS, 484, 4337

Tyson, J. A. 2000, Gravitational Lensing by Clusters of Galaxies, ed. Murdin, P., art. ed. 2144

Tyson, J. A., Kochanski, G. P., \& Dell'Antonio, I. P. 1998, ApJL, 498, 107

Williams, L. L. R. \& Schechter, P. L. 1997, arXiv:astroph/9709059 and Astron.\& Geophys. 38, Iss. 5, 10

Winn, J. N., Rusin, D., \& Kochanek, C. S. 2004, Natur, 427,613

Wise, J. H. 2019, arXiv:1907.06653

Wittman, D. M., et al. 2000, Natur 405, 143

Wu, X. 2008, Talk at the Summer School on Cosmic Reionization at the KIAA-PKU, Beijing, China

Yang, J., Wang, F., Fan, X., et al. 2019, AJ, 157, 236

Zaroubi, S. 2013, in The First Galaxies, ASSL, 396, 45 